\title{
La seguridad alimentaria en Caldas, Colombia. Un ejercicio de implementación de política pública
}

\section{The safe food in Caldas, Colombia. An practice of implementation of public policy}

Aceptación: 9 de marzo de 2010.

Sandra Milena Franco Patiño ${ }^{1}$

Aprobación: 30 de mayo de 2010.

\section{RESUMEN}

El derecho a la alimentación, como un derecho inherente a la condición humana, requiere trascender su reconocimiento en el plano legal/formal de los derechos hacia la materialización efectiva de los mismos, mediante la conjugación de acciones gubernamentales, institucionales y comunitarias. Este artículo se propone evidenciar que una implementación eficiente de la política pública en Seguridad Alimentaria y Nutricional, SAN, debe asumir la perspectiva de la titularidad del derecho a la alimentación en sus acciones, estrategia central para abordar integralmente la inseguridad alimentaria, no solo desde la carencia o la expresión de condiciones de pobreza, como generalmente se ha hecho. Tal planteamiento es resultado de las reflexiones generadas en el marco del proyecto Fortalecimiento del capital social y humano para la SAN, en Caldas (Colombia), desarrollado como parte de la puesta en marcha de la política pública en SAN con grupos familiares y comunitarios en seis municipios del departamento.

Palabras clave: Derecho a la alimentación, seguridad alimentaria y nutricional, política pública.

\begin{abstract}
The right to food as an inherent to the human condition right requires transcend its recognition in the legal and formal drawing rights towards the effective realization of them, using the conjugation of governmental, institutional and community actions. This article intends to show that food insecurity is not only the expression of poverty - as he has generally been understood-, but a multicause social fact that requires to be comprehensively addressed from the perspective of ownership of the right to food. Such an approach is based on reflections generated within the framework of the project "strengthening of the social and human capital for food and nutritional security (SANs)" as part of implementing the SAN Policy Department of Caldas (Colombia).
\end{abstract}

Docente investigadora del Departamento Estudios de Familia, Universidad de Caldas (Colombia).

Sandra.franco@ucaldas.edu.co. Doctoranda en Ciencias Sociales (FLACSO- Argentina). 


\section{Introducción}

El derecho a la alimentación es un derecho inherente a la condición humana. Paradójicamente, los logros en las condiciones de desarrollo de los países del llamado 'tercer mundo' parecen estar cada vez más distantes de garantizar a la mayoría de la población las oportunidades de acceso a alimentos adecuados en cantidad y calidad.

Los datos de la Organización de las Naciones unidas para la Agricultura y la alimentación, $\mathrm{FAO}^{2}$, indican que en el último decenio (1995-97/ 2004-06) el número de personas subnutridas en el mundo mantuvo un crecimiento lento pero constante. Ese número habría aumentado a 1.020 millones durante el $2009-100$ millones de personas más respecto al 2008-, lo que significa que casi una sexta parte de la humanidad padece hambre, una de las cifras más elevadas desde la crisis alimentaria de 1970.

El incremento constante en el número de personas que padecen hambre en el mundo desde la década de 1990 a la fecha ha puesto de manifiesto uno de los flagelos más dramáticos de las sociedades actuales. Para enfrentarlo, la ONU y sus Estados miembros han definido políticas y medidas vinculantes que permitan hacer efectivo el derecho a la alimentación, reconocido en la Convención de los Derechos Humanos como derecho esencial para la vida de todas las personas. Sin embargo, las acciones y las inversiones en esta materia parecen ser insuficientes, al atender las consecuencias más que los factores estructurales que lo generan.

Pese al fracaso de las medidas adoptadas por los gobiernos en las diversas naciones para reducir el problema del hambre, es innegable la necesidad de mantener el com- promiso con la garantía de los derechos fundamentales de los seres humanos y avanzar en el alcance de este propósito. Esto implica trascender el reconocimiento legal de los derechos a la materialización efectiva de los mismos y, para ello, la acción gubernamental, institucional, familiar y comunitaria constituye uno de los mecanismos para hacerlo posible.

En Colombia, un país rico en recursos naturales, la mayoría de la población es víctima de la pobreza y el hambre. La información registrada por el Departamento Nacional de Estadística, DANE, en 2005, señala que la pobreza ha crecido en los últimos años hasta situarse en $66 \%$ de la población; $49,2 \%$ de las personas se ubica por debajo de la línea de pobreza y $14,7 \%$ en indigencia. En materia nutricional, "las cifras oficiales indican que 12 de cada 100 niños y niñas menores de 5 años sufren de desnutrición crónica, 5,4\% desnutrición global y el 1,1\% desnutrición aguda; el $44,7 \%$ de las mujeres gestantes son anémicas o el $11 \%$ dan a luz bebés con bajo peso; el $53 \%$ de los menores de 6 meses de edad no reciben lactancia materna exclusiva; el 36\% de la población tiene una deficiente ingesta de proteínas y, el $41 \%$ del total de hogares colombianos manifiesta algún grado de inseguridad alimentaria (Comisión de Derechos Humanos, ONU: 2007)".

Ante esta abrumadora realidad, el gobierno colombiano institucionalizó en el año $2005^{3}$ la Política de Seguridad Alimentaria y Nutricional $^{4}$ (en adelante SAN), de obligatorio cumplimiento en los niveles nacional, departamental y municipal, para "garantizar que toda la población colombiana disponga, acceda y consuma alimentos de manera permanente y oportuna, en suficiente cantidad, variedad, calidad $e$ inocuidad" (CONPES, 2008:28). En este marco, los gobiernos depar-

2 El Estado de la Inseguridad Alimentaria en el mundo 2009. Crisis económicas: repercusiones y enseñanzas extraídas. Disponible en http://www.fao.org/docrep/012/i0876s/i0876s00.htm, consultado octubre 16 de 2009.

3 Aunque se institucionaliza una medida política en el marco de relaciones políticas internacionales, a lo largo del $\mathrm{S} X \mathrm{X}$ en el país se han desarrollado diversos programas para enfrentar el problema de alimentación y nutrición de la población, articulado con las áreas de salud, educación y desarrollo rural a través de los distintos Ministerios.

4 Documento Consejo de Política Económica y Social CONPES, No. 113, marzo de 2008. Esta política recoge gran parte del proceso adelantado por el Plan Nacional de Alimentación y Nutrición, 1996-2005, antecedente fundamental de estas acciones. 
tamentales y municipales definen e implementan lineamientos de política acorde con las realidades particulares del contexto local y establecen alianzas institucionales para la ejecución de programas y proyectos que operativicen la política.

En este escenario, a partir del año 2006 Caldas $^{5}$ (Colombia) lidera, en cabeza de la Dirección Territorial de Salud ${ }^{6}$ y la Red Departamental de Seguridad Alimentaria, la formulación de la política SAN departamental (2007-2017) y la construcción de los planes locales en los 27 municipios del Departamento. Definida la política pública, impulsó su implementación en los municipios con mayor desnutrición crónica en menores de cinco años. Para ello, la Dirección Territorial de Salud establece un convenio interinstitucional ${ }^{7}$ con el programa académico de Desarrollo Familiar $^{8}$ de la Universidad de Caldas para ejecutar el proyecto Fortalecimiento del capital social y humano para la seguridad alimentaria y nutricional en Caldas, como una de las acciones contempladas en las líneas de política del Plan Departamental SAN .

El proyecto contenía dos énfasis centrales: el primero, orientado al componente sociocultural de las familias y las comunida- des para identificar las concepciones que soportan sus prácticas (acceso, transformación y consumo) alimenticias, así como los recursos disponibles para potenciar la producción de alimentos. El segundo, referido al componente institucional, del que disponen las administraciones municipales para la puesta en marcha de los planes locales de seguridad alimentaria, para establecer acciones de acompañamiento que fortalecieran la capacidad de gestión local.

La experiencia de trabajo con familia $a^{10}$, llevada a cabo en los municipios de Riosucio (Resguardo indígena Cañamomo, Lomaprieta y La Montaña) y Supía (veredas Palmasola, la Playita y Mudarra), de la subregión ${ }^{11}$ Alto Occidente Caldense, desde el año 2006, fue el antecedente para formalizar las acciones institucionales, mediante objetivos comunes de impulso a la política. Así, en el año 2008 se inicia una prueba piloto del proyecto en seis municipios de Caldas ${ }^{12}$ : Supía, Marquetalia, Norcasia, Salamina, Villamaría y Belalcázar (anexo no. 2); en el 2009 se extiende a 7 municipios más, para un total de 13 municipios atendidos hasta la fecha.

El conocimiento y el aprendizaje obtenidos en el marco de este trabajo interinsti-

5 El Departamento de Caldas se encuentra ubicado en el centro occidente del país (Ver anexo No.1, ubicación geográfica del departamento), dividido administrativamente en 27 municipios, con una población total de 1.172.510 habitantes, de los cuales 382.193 se concentran en su capital, Manizales.

6 La Dirección Territorial de Salud de Caldas es una dependencia de la Gobernación, con funciones administrativas y de coordinación en pro del desarrollo del sector salud y del sistema de seguridad social en el departamento, encargado de la asesoría, asistencia técnica, vigilancia y control del sistema de salud.

7 Convenio No. 00IC064, inscrito en la Vicerrectoría de proyección con el código 2010-192 para la Universidad y No.639 para la Dirección Territorial.

8 Desarrollo Familiar es un programa de formación académica en el nivel de pregrado, creado en 1983 en la Universidad de Caldas para conceptualizar científicamente las relaciones familia - sociedad en el marco del desarrollo. Para ello, habilita profesionales que trabajen con familias desde la investigación, la educación y la acción, para promover procesos de cambio familiar.

9 El proyecto se articula con los programas de la primera línea de acción “ingreso y generación de empleos" y la tercera línea "estilos de vida saludable".

10 El trabajo con familia corresponde al proceso de práctica durante la formación en Desarrollo Familiar. Este se realiza durante dos años continuos, en los cuales estudiantes y docentes se insertan en una zona geográfica específica para intervenir grupos familiares y comunitarios, mediante la investigación, la educación y la acción, en perspectiva de promover cambios tendientes a potenciar el desarrollo individual, familiar y social.

11 En el 2004, el departamento de Caldas utiliza la clasificación de los distritos agroindustriales para agrupar a los municipios en 6 subregiones (Bajo y Alto Occidente, Magdalena Caldense, Alto Oriente, Norte y Centro Sur) según características de población y vocación productiva, de manera que aumenten los niveles de competitividad mediante el afianzamiento de sus relaciones económicas, especialmente. El propósito de esta división es orientar los planes y programas de inversión y desarrollo en los municipios.

12 La selección de los municipios se hizo con base en los siguientes criterios: Un municipio por cada una de las subregiones en que está dividido el Departamento, que presenten las mayores tasas de desnutrición crónica en menores de cinco años, que hayan avanzado la formulación de los planes locales SAN y que existiera voluntad política en el tema. 
tucional permite plantear que una implementación eficiente de la política pública en SAN debe asumir la perspectiva de la titularidad del derecho a la alimentación en sus acciones. Desde tal perspectiva, la inseguridad alimentaria que afrontan las familias no es solo la expresión de condiciones de pobreza -como generalmente se ha entendido-, sino un hecho social multicausal que requiere acciones institucionales que, además de considerar integralmente el fenómeno de la inseguridad alimentaria, garanticen el derecho a la alimentación como pretensión universal de derecho humano.

Tal afirmación -que en principio parece obvia-, surge del análisis de las acciones gubernamentales e institucionales que fundamentan la gestión en el ámbito municipal de la política pública en SAN y busca, por un lado, visibilizar los aspectos socioculturales que fundamentan las prácticas de alimentación en las familias y los factores del entorno que aumentan las condiciones de riesgo alimentario. Entender el contexto sociocultural, económico y político en el que se encuentran inmersos los grupos familiares permite ampliar los referentes desde los cuales se hace lectura del problema alimentario, que desde la política pública suele estar asociado generalmente a la escasez de recursos económicos para la provisión de alimentos.

Por otro lado, se debe analizar el papel que le corresponde al Estado (institucionalidad, proceso, actores) en la implementación de políticas públicas que garanticen la titularidad de los derechos, en tanto las acciones concretas evidencian un distanciamiento entre el propósito de la política y su aplicación, por cuanto éstas suelen mitigar algunos factores de vulnerabilidad de las familias más que hacer efectivo la responsabilidad universal de garantía de derechos.

Para sustentar este argumento, este artículo expone la fundamentación interna- cional y nacional de los derechos, en particular, el derecho a la alimentación como marco de referencia desde el cual se aborda la discusión respecto a la Seguridad Alimentaria y Nutricional. Un enfoque de derechos reconoce que estos deben ser garantizados a todos los sujetos como condición básica de humanización, sin distinción de sexo, edad, condición social, religiosa, económica o de otro orden. No obstante, los propósitos del modelo de desarrollo económico neoliberal generan una contradicción discursiva entre el marco prescriptivo de los derechos (deber ser) y las acciones gubernamentales que asumen un carácter asistencial distanciado de la garantía del derecho fundamental a la alimentación que se propone la política.

Seguidamente, se describe parte de la prueba piloto realizada en los municipios de Caldas, referida a las concepciones socioculturales de las familias en torno a las prácticas de alimentación y algunas reflexiones respecto de los alcances y restricciones de la implementación de la política pública SAN en el ámbito local. Los informes de campo de las estudiantes/profesionales dan cuenta de las acciones educativas adelantadas con las familias y la comunidad, mediante visitas familiares y reuniones comunitarias desarrolladas entre los meses de agosto y diciembre del 2008, en inmersión completa en el contexto local ${ }^{13}$, los cuales constituyen el referente empírico de este artículo.

\section{El derecho a la alimentación}

"Conozco el hambre, la sentí... esa hambre está en mí. No puedo olvidarla”.

$$
\text { (J.M.G. Le Clézio) }
$$

El derecho a no tener hambre se reconoció en el artículo 25 de la declaración Universal de los Derechos Humanos (ONU, 1948). En él se que indica: "Toda persona tiene derecho a un nivel de vida adecuado que 
le asegure, así como a su familia, la salud y el bienestar, en especial la alimentación...”. Su carácter vinculante se reconoció en el artículo 11 del Pacto por los Derechos Económicos, Sociales, Políticos y Culturales, DESC (1966).

Los derechos consagrados en esta declaración con carácter universal, inviolable e inalienable, sostienen la interrelación existente entre los derechos civiles, políticos, económicos, sociales y culturales en un mismo plano de importancia. Sin embargo, la reflexión jurídica y la acción política han otorgado primacía a los derechos civiles y políticos (DCP) y han sido en muchos sentidos objeto de mayor atención, codificación jurídica e interpretación judicial (CEAM, 2004); mientras que los DESC son considerados derechos de 'segunda clase', con escaso conocimiento y divulgación entre la población.

Una de las razones que se aducen para esta diferenciación es que los DESC exigen disponer de abundantes recursos económicos para su cumplimiento, al ser 'obligaciones positivas' (autorrealización de los individuos) que exigen que el Estado tome medidas para alcanzarlos, por lo cual su efectividad se alcanzará de manera progresiva en el tiempo; en cambio, la garantía de los DCP se consideran 'obligaciones negativas' (ausencia de interferencia por parte de terceros) en las que el Estado debe abstenerse de tomar medidas que impidan a la gente ejercer sus derechos y proceder a su práctica inmediata, cuando los ciudadanos interpongan recursos ante los órganos del Estado toda vez que estos hayan sido violados (Merchán C, 2007).

Esta situación ayuda a entender -en parte- por qué después de 60 años de explicitación jurídica del derecho a la alimentación, todavía millones de personas en el mundo mueren de hambre. El derecho a la alimentación se considera un derecho de segunda generación, aspecto bastante discutible si se tiene en cuenta que sin la satisfacción de esta necesidad físico-biológica no es posible la vida humana, y el despliegue de capacidades psicológicas y sociales, como tampoco el disfrute de otros derechos; que asocia para su cabal cumplimiento, acciones en diversas esferas de la vida social: productiva, económica, educativa, ambiental, sanitaria y cultural.
Para avanzar en el significado de este derecho y las obligaciones que de él se derivan, fue decisivo el compromiso político de la FAO a partir de las Cumbres Mundiales de Alimentación (1996 y 2001), en las que se impulsan acuerdos y medidas orientadas a superar la distancia entre el reconocimiento legal de este derecho y la realización plena del mismo; hasta el punto que el Comité de los DESC encargado de hacer seguimiento y vigilancia a los acuerdos del Pacto por los DESC en 1999 estipula en la observación general No. 12 la responsabilidad de los gobiernos para garantizar y hacer posible que las personas puedan producir o comprar alimentos suficientes (CEAM, 2004: 24).

En consonancia con las obligaciones contraídas en la Cumbre Mundial de la Alimentación de 1996, Colombia formula el Plan Nacional de Alimentación y Nutrición, PNAN, 1996-2005, con el objetivo de contribuir al mejoramiento de la situación alimentaria y nutricional de la población colombiana (Ministerio de Protección Social, 2005). Los resultados de la evaluación de los alcances y las dificultades de la implementación de esta política realizada en el año 2003 fueron el soporte para avanzar en la construcción de una política de Estado que definiera un marco general de acción para las entidades públicas y privadas del país en esta materia.

Así, en el año 2005 se formula la política de Seguridad Alimentaria y Nutricional (policy) que materializa la pretensión política (polity) de la Constitución Nacional de 1991, que establece como deberes del Estado el derecho a la alimentación equilibrada, como un derecho fundamental de los niños (artículo 44 ), y la producción de alimentos (artículos $64,65,66)$.

El objetivo de la política SAN es "garantizar el derecho a la alimentación a la población colombiana, especialmente la que se encuentra en situación de inseguridad alimentaria y nutricional, para que disponga, acceda y consuma alimentos de manera permanente y oportuna, en suficiente cantidad, variedad, calidad e inocuidad" (Política SAN, 2005).

Al analizar los objetivos de política expresados en la declaración de los Derechos 
Humanos, en el Pacto por los DECS, los lineamientos de la Cumbre de Roma y la política SAN, se aprecia que la garantía del derecho a la alimentación enfatiza en la disponibilidad y el acceso a los alimentos, más que en la titularidad del mismo. Es decir, el problema del hambre y la desnutrición que se vive actualmente en el mundo es el resultado de la pobreza y la inequidad frente a la distribución de la riqueza -que deja por fuera a un grupo considerable de consumidores que no cuentan con la capacidad económica para adquirir los productos básicos para satisfacer sus requerimientos alimenticios-, más que la incapacidad tecnológica para garantizar la oferta de alimentos. Por lo tanto, la seguridad alimentaria no depende tanto de la disponibilidad de alimentos existentes en el mercado como del acceso efectivo que las personas y las familias tienen a ellos, que no se restringe exclusivamente a los ingresos económicos o las posibilidades de producción, sino también a las dotaciones iniciales, las condiciones, las oportunidades, las capacidades y los recursos que poseen las personas y las familias para ello.

Las titulaciones, entendidas como "la totalidad de las cosas que una persona puede tener en virtud de sus derechos" (Sen, 2002: 18), van más allá del derecho a estar libre de hambre. Implica considerar las diversas oportunidades y los diversos bienes asociados a tal derecho: ingreso y permanencia al sistema escolar, condiciones de salud, disponibilidad de un empleo, reposición de fuerza de trabajo, propiedad sobre la tierra, participación en la vida social, disfrute del tiempo de ocio, entre otros. Es, por tanto, mucho más que la satisfacción de necesidades en torno a la comida. Esta solo se constituye en una pequeña parte de la solución, pues son los problemas de carácter estructural los que deben vincularse a las decisiones y al trabajo en torno al derecho a la alimentación y nutrición que hagan posible la titularidad del mismo.

En términos de Sen, el derecho a la alimentación exige comprender las causas estructurales del problema en relación con el poder económico y la libertad fundamental que los individuos y las familias poseen para conseguir la propiedad sobre los alimentos (por producción o compra en el mercado), "en lo que tenemos que centrar la atención no es en las existencias totales de alimentos que hay en la economía, sino en el 'derecho económico' de que disfruta cada persona: los bienes sobre los que puede demostrar su propiedad y control" (1999:201).

En tal sentido, las medidas políticas que los gobiernos definen para garantizar el derecho a la alimentación desde una perspectiva de la titularidad de derechos requieren considerar estas en relación con el modelo económico y político de la sociedad. En Colombia, al igual que en diversos países, el modelo de desarrollo económico neoliberal instaurado resulta incompatible con el enfoque de titularidad y garantía del derecho a la alimentación -tal y como puede apreciarse en las cifras y los logros obtenidos a la fecha en este campo-, debido a que la teoría del libre comercio lo reduce a los mecanismos de acceso a los bienes y servicios ofrecidos y regulados por el mercado, bajo un sistema de producción capitalista que dispone de una capacidad infinita de oferta a la que no corresponde una igual demanda.

En esta lógica, la inseguridad alimentaria se concibe como un problema de disponibilidad y acceso a los alimentos, como bien se aprecia en los objetivos de la política, más que un problema económico y político de las naciones, como efectivamente lo es. Esto es así porque los regímenes y sistemas políticos de nuestras naciones, al adoptar tal modelo de desarrollo, comparten los intereses propuestos; en consecuencia, no existe un pensamiento ni una acción crítica respecto al desarrollo del país, pues la hegemonía del modelo pareciera indiscutible e irreversible.

Un Estado que considere el problema de la alimentación y la nutrición de su población como prioridad y eje del desarrollo debe pensar y diseñar una política que considere la soberanía alimentaria como alternativa a las políticas que impone el modelo neoliberal. Es decir, decidir autónomamente su producción de alimentos por encima de la lógica del mercado, asegurar que estos se produzcan en el territorio nacional $y$, sobre todo, que estén disponibles para la gente, procurando que dicho suministro proteja la autonomía alimentaria de los pueblos (Foro Social Mundial, 2004). 
Simultáneamente a ello, debe desplegar un sistema de seguridad y protección social que posibilite a la población oportunidades y el despliegue de capacidades que les permita asumirse como sujetos titulares de derechos, con deberes y obligaciones en el ejercicio de los mismos, un sistema que ofrezca condiciones y oportunidades para que los individuos y las familias tengan su capacidad de libertad para optar y decidir.

Pese a este marco prescriptivo, el gobierno colombiano, en sus diferentes niveles de actuación (nacional, departamental, municipal), ha asumido la seguridad alimentaria como marco desde el cual se garantiza el derecho a la alimentación. El concepto de seguridad refiere a la capacidad de todo sistema para estar libre de cualquier riesgo o peligro o también a la confianza en algo o alguien, la certeza de que algo va a cumplirse; pero ¿cómo garantizar a los ciudadanos la certeza de este derecho, cuando la política no está diseñada para garantizar los derechos fundamentales a todas las personas de una nación, sino que se focaliza en los más pobres y vulnerables, quienes, a razón del modelo han dejado de ser sujetos de derechos? La acción pública de los gobiernos es apenas complementaria con el mecanismo básico que establece el mercado como fuente principal para el bienestar y, en consecuencia, las acciones asumen un carácter compensatorio para los grupos sociales que no se insertan o no logran mantenerse en el mercado.

Se plantea la seguridad como una manera de evitar o reducir las condiciones de riesgo y vulnerabilidad que enfrentan las personas y las familias a tener hambre, o de otra manera, garantizar el derecho a la alimentación, pero bajo paliativos que si bien contribuyen a resolver situaciones concretas no logran ser sostenibles en el tiempo, como propósito de calidad de vida.

En coherencia con ello, la política define la seguridad alimentaria y nutricional como "la disponibilidad" suficiente y estable de alimentos, el acceso y el consumo oportuno y permanente de los mismos en cantidad, calidad e inocuidad, por parte de todas las personas, bajo condiciones que permitan su adecuada utilización biológica para llevar una vida saludable y activa" (CONPES, 2008:3).

Como puede apreciarse, la concepción política (polity) de esta política (policy) se ajusta a los objetivos del modelo de desarrollo económico instaurado y no a su transformación, por ello el énfasis puesto en la disponibilidad (stock de producción), actualmente suficiente en la amplia y variada oferta de alimentos en el mercado. Mientras, las posibilidades concretas de acceso para aquellos que han quedado fuera del sistema de relaciones mercantiles siguen siendo evaluadas con el parámetro de adecuación a los fines previstos (mercados), condición que los ubica y los mantiene en condición de vulnerabilidad al no responder eficientemente a tales propósitos.

En tal sentido, la política no contempla una garantía real del derecho a la alimentación desde un principio de universalidad, en tanto condición inherente de lo humano, sino que se estipula bajo criterios de focalización de aquellos a quienes las fuerzas del mercado han dejado fuera, sobre quienes deben tomarse medidas para incluirlos nuevamente en el sistema.

Dicho de otro modo, las medidas políticas que el gobierno colombiano plantea para superar la inseguridad alimentaria distan mucho de ofrecer oportunidades y condiciones para garantizar el derecho a la alimentación del conjunto de la población. Estas se orientan a los sectores poblacionales que, en virtud de sus carencias o necesidades básicas insatisfechas, no logran insertarse adecuadamente al modelo, por ello, las ayudas (bien sea en dinero o especie) buscan 'paliar' situaciones específicas que, al ser superadas, permitan que aquellos que se quedaron al margen retornen al rebaño del que no debieron salir. No hay una pos-

14 Las negrillas son mías. 
tura crítica al sistema político y económico que haga posible entender y dimensionar las causas estructurales del fenómeno y, en consecuencia, soluciones substantivas que permitan superarlas, sino que la reproducción de un sistema hegemónico del que pareciera no existir retorno.

Pese a estas consideraciones críticas del enfoque político asumido en la política de SAN, no se desconocen los aportes que desde la acción concreta de las instituciones, como la Universidad, puede hacerse para favorecer el logro paulatino de este derecho. De ahí que el programa de Desarrollo Familiar de la Universidad de Caldas haya establecido un convenio de cooperación con el gobierno departamental para implementar acciones que, en el marco de la política Departamental y Nacional de SAN, contribuya a entender y dimensionar las realidades concretas de las familias y las comunidades que carecen de este derecho y, a su vez, contribuir a generar cambios y transformaciones en prácticas cotidianas concretas de los actores locales que hagan del derecho a la alimentación más que una prescripción una forma de vida.

2. La gestión pública: Un enfoque para la implementación de la política pública de SAN en Caldas

Como se indicó anteriormente, la política nacional de Seguridad Alimentaria y Nutricional es el marco orientador de las acciones que se desarrollan en el nivel departamental y municipal, para lo cual se formulan Planes Locales de Seguridad Alimentaria, en los que se especifican las condiciones alimenticias y nutricionales de la población en contextos concretos. Con base en sus realidades, se establecen programas y proyectos que contribuyan a superar la inseguridad alimentaria $y$, de esta forma, cumplir con los propósitos de la política nacional en lo local.

A continuación se exponen las acciones desplegadas en el proyecto, en sus dos componentes centrales, sociocultural e institucional, analizadas a la luz del enfoque de gestión pública, como enfoque de implementación de política en el que se sustentó el proyecto. A manera de contextualización, se muestran unas generalidades de los municipios relativas a la población, condiciones productivas y los principales programas de atención en materia de alimentación y nutrición. Seguidamente, se describen algunas concepciones socioculturales que las familias beneficiarias poseen respecto a las prácticas alimenticias y la capacidad institucional de las localidades para la gestión de la política pública SAN.

\subsection{Enfoque de la gestión pública}

La implementación de políticas públicas es mucho más que un problema administrativo o técnico de 'simple' ejecución; es un problema político relativo a la concreción de hechos a través de los cuales se espera construir una nueva realidad que supere la actual. Concebido de esta forma, la implementación de política es mucho más que la puesta en marcha de lineamientos definidos en la normatividad o reglamentación reguladora de objetivos y acciones específicas, es fundamentalmente un escenario de lucha y confrontación política, un juego de intereses en el que se develan relaciones de poder político, económico y social.

Con esta posición, el proyecto de fortalecimiento del capital social y humano para la SAN en Caldas asumió el enfoque de gestión pública en el acompañamiento técnico que brindó a las instancias administrativas de gestión y decisión políticas de las localidades, de manera que fortalecieran y aumentaran su capacidad institucional para garantizar el derecho a la alimentación, a la vez que se hacía lectura de las complejas redes, tensiones, idas y vueltas surgidas a lo largo del proceso de acompañamiento en la implementación de la política en algunos de sus programas.

El enfoque de gestión pública ha sido pensado y destinado para los diseñadores y evaluadores de política, encargados de estructura procesos óptimos para la ejecución efectiva de los principios y objetivos establecidos por la ley o el programa. Para garantizar y maximizar las probabilidades de logro de los objetivos, algunos autores como Sabatier y Mazmanian (1979) han propuesto cinco condiciones básicas necesarias de tener en cuenta 
para la implementación efectiva, eficaz y eficiente de las políticas. Estas son ${ }^{15}$ :

a) El programa debe disponer de una teoría sólida relativa al cambio de comportamiento de las personas necesarias para alcanzar los objetivos.

b) La reglamentación (ley, norma, decreto) debe contener directrices claras y precisas que estructuren el proceso de implementación, de manera que se maximicen las posibilidades de obtener el cambio esperado.

c) Los decisores de política, al igual que los técnicos, profesionales y demás líderes encargados de su implementación, deben disponer de capacidades políticas y de gestión.

d) El programa debe disponer de apoyos activos para su ejecución.

e) La prioridad de los objetivos va más allá de pretensiones particulares de gobierno.

Una lectura exhaustiva de la política SAN a la luz de estas condiciones permite decir que la teoría de cambio social implícita es que, si hay alimentos suficientes en cantidad y calidad y si se promueven opciones para que los individuos y las familias dispongan, accedan y los consuman en forma oportuna y adecuada, entonces será posible garantizar una vida saludable y activa.

Sobre esta relación de causalidad desde la que se explica el problema alimentario existe, en principio, cierto consenso entre técnicos, diseñadores de política, académicos, instituciones y comunidad. Ahora bien, el punto de discusión está en los medios que se utilizan para hacer efectivo este propósito. En la lógica gubernamental - estatal, los medios están centrados en el mercado; en consecuencia, un grueso de los programas previstos para que las familias puedan alcanzar una canasta de alimentos, buscan dotarlas de condiciones y recursos propios de la oferta y la demanda. Aquí el problema se resuelve mediante el suministro de una dieta alimentaria básica que sostenga la vida.

En la lógica comunitaria no gubernamental, los medios están centrados en la capacidad del Estado para producir alimentos respetando los saberes, las tradiciones, la diversidad y el manejo adecuado del medio ambiente, con subsidios y programas que favorezcan a los agricultores; distribuirlos equitativamente al conjunto de la población de manera que todos puedan acceder en igualdad de condiciones a alimentos de buena calidad. Aquí, el problema se resuelve fortaleciendo los sistemas políticos para que, además de corregir las deficiencias de la mano invisible del mercado, procuren un desarrollo soberano de la nación. Este último aspecto es el punto álgido y conflictivo del debate. Por lo pronto, es necesario e imperativo hacer parte de las acciones existentes y tratar de promover diálogos y encuentros entre los diversos actores, para avanzar en el consenso de un proyecto de desarrollo en donde las diversas posturas se encuentren representadas.

\subsection{Generalidades de los municipios beneficiarios}

El punto de partida fue conocer las condiciones de producción de los municipios y la intervención gubernamental e institucional para la garantía del derecho a la alimentación ${ }^{16}$.

Respecto a la población de los municipios, en la Tabla No. 1 se aprecia que tres de los municipios participantes del proyecto concentran su población mayoritariamente en el área rural y tres disponen de una población mayoritariamente urbana. En el caso de Norcasia, el bajo número de habitantes obedece

15 Lo esbozado aquí es parte de lo reseñado por Roth (2002: 115).

16 Las fuentes de información para fundamentar el diagnóstico fueron: los planes de desarrollo (departamental y municipal); planes locales SAN, planes de acción de las secretarías encargadas de implementar el plan SAN en cada municipio; revisión de informes disponibles en las diversas instituciones del municipio, entrevista a funcionarios de las UMATAS o Centros Provinciales de Gestión Agropecuaria, Secretarios de despacho de la administración municipal, del Instituto Colombiano de Bienestar Familiar ICBF y de Comfamiliares. La disparidad en la información y en algunos casos la inexistencia de ellas dificultó contar con criterios generales al momento de sistematizar. 
a que este era un corregimiento del municipio de Samaná hasta el año 1999, momento en el cual se erige como municipio, mientras que Villamaría tiene un número mayor de habitantes, debido a la cercanía con la capital del Departamento, Manizales, lo que posibilita la movilidad e integración permanente con los bienes y servicios de los que dispone la ciudad capital. Este hecho ha permitido que después de Manizales, sea el municipio más industrializado del departamento.

Al ser Caldas uno de los Departamentos de la zona cafetera, la mayoría de sus municipios tienen fundada su economía en la producción del grano que, hasta el rompimiento del pacto de cuotas en 1989 , constituyó el producto base de la economía colombiana. Asociado con el cultivo del café se encuentra el plátano, utilizado como sombrío, base de la alimentación tradicional especialmente en las zonas rurales. También es significativa la producción de caña panelera cultivada de manera tradicional, especialmente en el municipio de Supía, donde ocupa el primer renglón económico. Allí se producen aproximadamente 4.900 toneladas de panela al año, con 1.400 hectáreas de caña sembrada y 560 trapiches comunitarios. En Salamina se producen 1.459 toneladas de caña panelera al año en un área de 575 hectáreas.
En orden de importancia, la ganadería ocupa el segundo renglón de actividad económica de los municipios, especialmente en Salamina (Corregimiento de San Félix) con 25.000 cabezas de ganado, Villamaría y Norcasia. En este último, la ganadería se presenta como un renglón promisorio ante la proximidad del municipio al Magdalena medio, reconocido como zona que abastece de carne a una amplia región del occidente colombiano.

En relación con los programas de Seguridad Alimentaria y Nutricional, los municipios han dado prioridad a la atención de la primera infancia a través del programa Atención Integrada a las Enfermedades Prevalentes de la Infancia, AIEPI; madres gestantes y lactantes, crecimiento y desarrollo y programas de promoción y prevención coordinados con las entidades de salud, el Instituto Colombiano de Bienestar Familiar, ICBF, y Confamiliares ${ }^{17}$. Ligado a esto, se enfatiza en el apoyo a la producción agrícola, base fundamental de la economía, especialmente a través de las UMATAS (Unidades Municipales de Asistencia Técnica y Agropecuaria), que promueven la organización de las comunidades y el fortalecimiento de las asociaciones de productores ya existentes, generalmente mediante la provisión de insumos, asesoría técnica y, en algunos casos, subsidios o créditos a la producción.

Tabla No 1

Población total de los municipios diferenciado por zona urbana y rural

\begin{tabular}{|l|c|c|c|c|c|c|}
\hline Población & Belalcázar & Supía & Salamina & Marquetalia & Norcasia & Villamaría \\
\hline Urbana & 4.842 & 11.573 & 11.206 & 5.646 & 4.128 & 35.772 \\
\hline Rural & 6.485 & 12.499 & 7.075 & 8.234 & 2.395 & 9.266 \\
\hline Total & 11.327 & 24.072 & 18.281 & 13.880 & 6.523 & 45.038 \\
\hline
\end{tabular}

Fuente: Censo General de Población del DANE 2005.

17 El ICBF es la entidad gubernamental encargada de la atención a los derechos de los niños, niñas y familias en Colombia, dependiente del Ministerio de Protección Social tiene cobertura en todo el país. Confamiliares es la Caja de Compensación Familiar, creada en 1960 para otorgar beneficios a los trabajadores formalmente vinculados a empresas. Las cajas de compensación son de obligatorio cumplimiento de los patronos a los trabajadores y asumen nombres diversos según la región en que se ubiquen. 
En cuanto a ayudas alimenticias, se destaca los programas del ICBF: Madres FAMI, hogares comunitarios, restaurantes escolares, escenarios institucionalizados que, además de ofrecer alimentación a niños, niñas, adolescentes y jóvenes, adelanta procesos educativos relativos a la crianza y cuidado de los niños y las niñas, conforme a los derechos de la infancia. Asimismo, las administraciones municipales ofrecen ayudas alimenticias (bien sea con alimentos o dinero para la compra de estos) a los adultos mayores.

\subsection{Concepciones socioculturales de las familias de las prácticas de alimentación}

El trabajo con familias, comunidades y grupos asociativos se focalizó en veredas o barrios $^{18}$ identificados como de mayor vulnerabilidad por condiciones de pobreza, con los cuales se adelantaron procesos educativos para reconocer las prácticas de alimentación familiar y las implicaciones que ello tiene para el desarrollo individual, familiar y social. El proceso educativo se articuló a las acciones de producción de alimentos, mediante el montaje o adecuación de huertas caseras o huertas comunitarias. La UMATA ofrece las semillas y el acompañamiento técnico y las familias y comunidades, con su fuerza de trabajo, realizan el montaje y mantenimiento de las mismas.

En cuatro de los seis municipios se promovió la siembra de alimentos de autoconsumo en la huerta casera, escolar y comunitaria. Paralelamente, mediante visitas familiares se hizo una aproximación al proceso de alimentación que se vive en las familias y en particular, la forma como este interviene en el estado nutricional de infantes. En el caso del municipio de Villamaría, el énfasis educativo estuvo puesto en la organización y la participación comunitaria, mediante el trabajo con asociaciones de productores de hortalizas y verduras, en lo relativo a la comercialización de los productos y el mejoramiento de la calidad y la inocuidad de los mismos.
En Belalcázar, la estrategia AIEPI demandó un trabajo familiar centrado en prácticas de convivencia que favorecieran ambientes de vida saludable y reducción de la violencia intrafamiliar.

La importancia del ámbito familiar en los procesos de alimentación se respalda en estudios de orden mundial, en los que plantean que este se refiere a los procesos y dinámicas particulares que son propias de la vida familiar, los cuales "se generan por los vínculos que se crean a partir del nacimiento de un nuevo ser" (Kingsley D, 1930). Por tanto, tales procesos son diferentes a los que ocurren en otros ámbitos de vida en los que se desenvuelven los sujetos. Al respecto, agregan que "el nacimiento es un fenómeno, esencial y universal que es mucho más que un proceso biológico o social, porque involucra de modo fundamental, un número de procesos complicados interpersonales, mentales, emocionales, sexuales, genéticos, hormonales, temporales, espaciales, generacionales, experienciales y de desarrollo, que están entretejidos..." (Burr et al., 1988).

Con el nacimiento de cada integrante, las familias crean su propio ámbito y afrontan procesos complejos para el sostenimiento de la vida, con los que tratan de responder a los requerimientos humanos de su desarrollo, los cuales dependen de la alimentación y de las condiciones en que esta se dé.

La alimentación es entendida como un proceso cotidiano de orden biológico, psicológico y sociocultural, constituido por una serie de prácticas de transformación de los alimentos, entre las que son visibles las de elección, compra, preparación, servir, comer, aseo de alimentos, de utensilios y manejo de residuos. Para su realización, estas prácticas se basan en conocimientos, percepciones, habilidades y relaciones (afectivas, de poder, sociales, económicas) al interior de cada familia y con el medio comunitario y local, dado que son influenciadas por factores que las determinan en gran proporción, en tanto otros

18 Las veredas y los barrios corresponden a la división territorial de los municipios, las primeras referidas a las zonas rurales y los segundos a la zona urbana. 
emergen de las múltiples decisiones cotidianas (consientes o no) que toman las familias para llevarlas a cabo.

Es decir que, en cada ámbito familiar se construyen diferentes maneras de afrontar el proceso alimentario de sus integrantes en su conjunto y de cada uno de ellos, en respuesta a la particularidad del momento de desarrollo. A medida que transcurre, se entrecruzan las influencias con ámbitos externos, el comunitario y el local, regidas por las condiciones humanas y materiales de orden económico y sociocultural y las estrategias no siempre compatibles, de unos y otros, para mejorar el nivel de vida. En este proceso, el objetivo del desarrollo es la obtención de mayores niveles de seguridad alimentaria, de modo tal que se eviten los estados de desnutrición en infantes y niños.

En cuanto a los significados más comunes entre las familias rurales atendidas en los diferentes municipios de manera general, se aprecia lo siguiente:

En cuanto a la compra de alimentos, algunas madres destacaron que ellas son las encargadas de ir a comprar o mercar los alimentos que ellas creen necesarios, por ser ellas quienes saben qué comprar porque generalmente son las responsables de preparar los alimentos, lo cual muestra que las mujeres asumen un papel preponderante en la alimentación del grupo familiar; sin embargo, no puede obviarse el hecho de que el manejo del dinero es restringido, porque la cantidad total es aportada por el esposo, quien lo gana como producto de su trabajo, quien tiene mayor influencia en la decisión final de qué productos comprar, lo que incluye la restricción que se genera por la relación entre el monto de dinero, el costo de los mismos y la cantidad de alimentos que requiere la familia, según el tamaño y necesidades de sus miembros.

En cuanto al papel de algunas de las madres en la compra de alimentos, hay percepciones importantes sobre el conocimiento y administración de los alimentos derivado de sus experiencia, relativas a que les gusta ir a mercar porque hacen rendir la plata, ellas (madres, mujeres) saben qué alimentos nece- sitan comprar y cuáles son aptos para todos los integrantes de la familia. En las familias se utilizan varias estrategias para hacer rendir el dinero. Una de ellas es hacer el mercado con una periodicidad quincenal, para ahorrar los gastos de desplazamiento al pueblo donde generalmente acceden a los alimentos básicos.

En las prácticas de compra de alimentos entre familias, se aprecia que en su mayoría es realizada por las madres, aunque son sus esposos quienes les dan el dinero, ya que ellos hacen trabajo remunerado, mientras ellas hacen trabajo no remunerado, al encargarse de quehaceres domésticos. Estas labores ya no son aceptadas sin cuestionamiento por muchas madres, ante lo que manifiestan que "ver a un hombre metido en la cocina es algo muy extraño". Al ser la mujer la encargada de la compra de los alimentos, genera de manera directa o indirecta que se le atribuya cierto poder en la toma de decisiones, y aunque no es una situación relevante, muestra un avance hacia lograr un reconocimiento meritorio al papel de la mujer dentro de la familia.

En la elección de alimentos se evidencia que la preferencia por todos los alimentos para la preparación de comidas típicas tradicionales, como sancocho y frijoles, la realizan las madres y abuelas. Muchas de ellas coincidieron que estos dos platos son muy importantes y básicos al momento de alimentar a los integrantes del grupo familiar. La frecuencia de este hábito alimenticio puede estar asociado con la facilidad de acceso al plátano, la yuca, el frijol, la cebolla, el tomate, el cilantro, el aguacate y el banano, entre otros alimentos típicos de la región, que además son los que se cultivan en las huertas. Esta preparación, además de ser costumbre, también representa una mayor economía a la hora de acceder a estos alimentos.

En cuanto a los recursos económicos para la compra de alimentos, se encontró que estos son escasos, ya que las familias expresan que existen muchos alimentos que les gustaría adquirir con frecuencia y consumir con más regularidad: frutas, verduras, chocolate, leche, huevos, pan, pescado, pollo, yogur y queso, pero, “... no hay casi recur- 
sos económicos para comprarlos y no porque no nos gusten sino porque no alcanza para eso", más aún si se tienen en cuenta que estos alimentos son demasiado costosos en el mercado, lo que restringe las posibilidades de acceso ante los escasos ingresos. Asimismo, aunque respetan la preferencia de alimentos como los granos, también los prefieren por escasez de recursos económicos, porque rinden más al prepararlos.

Otro recurso para las familias es la propiedad de la tierra y su cultivo. Algunas cuentan con tierra y espacio para cultivar en huertas caseras y para la cría de animales, para el autoabastecimiento y para la venta.

Una práctica importante de destacar son las reglas que forman parte de las prácticas alimentarias, ya que estas generan de cierta manera organización y responsabilidades dentro del hogar. Los horarios que se manejan a la hora de comer están influidos por las arduas jornadas de trabajo de padres e hijos adultos, lo que otorga un carácter obligatorio. De igual forma, el estudio de los niños y las niñas también está sujeto a las jornadas de la institución escolar, lo cual influye en que haya pocos momentos en que todos puedan compartir los alimentos del día en familia (desayuno, almuerzo, comida).

Se presentan diferencias en los principios para decidir a quién se le sirve primero los alimentos. Algunas familias priorizan a los más pequeños, en otras están primero los hombres adultos y luego los niños y las mujeres y madres se sirven al final; esto deja ver, que aunque hay cambios, se conservan ideas tradicionales sobre el mayor reconocimiento del hombre-padre por su trabajo y en menor posición los niños y las mujeres, en particular, la madre, quien se relega a sí misma, y también se ve obligada, al no contar con el mismo tiempo para sentarse a disfrutar de los alimentos con la misma tranquilidad que lo puede hacer su esposo y demás miembros de la familia.
En la percepción de prácticas alimentarias de cuidado, se destacan los hábitos de manipulación de alimentos, ya que es evidente que las familias evitan problemas mayores en la salud, al hervir y filtrar el agua que no es potable. Se encontró que en las familias se hierve el agua antes de consumirla en cualquier forma, sin embargo, también hay casos en donde solo hierven el agua para preparar los alimentos de los niños y niñas, mientras que para los adultos no realizan dicho procedimiento. De igual modo, la mayoría de las madres expresan que ellas al momento de preparar los alimentos los lavan muy bien, se fijan bien que no estén dañados.

\subsection{La gestión pública en el ámbito local}

La aproximación y permanencia directa en los municipios y el contacto con los actores locales fueron relevantes para la concertación de las acciones a desarrollar con los técnicos responsables de la gestión política en la municipalidad.

Este trabajo se concentró en la dinamización de la red local SAN ${ }^{19}$, instancia que convoca a las instituciones gubernamentales, no gubernamentales y comunitarias del municipio, para debatir, definir y promover acciones articuladas en esta temática. El interés de la red es, por una parte, alcanzar la integración de proyectos y acciones entre las diversas instituciones, para aumentar la eficiencia y eficacia de estos, evitando la disgregación que ha caracterizado el desarrollo de las regiones y con ello, el uso y abuso en el trabajo comunitario. Por otra parte, se espera que esta instancia de participación alcance representación política en la medida que las decisiones estén vinculadas a planes y programas de gobierno ya institucionalizados, a través de los cuales se despliegan recursos y objetivos de desarrollo en el largo plazo; es decir, que los programas y proyectos SAN se correspondan e integren con las demás áreas del desarrollo. De esta manera institucionalizar el quehacer y la continuidad de procesos en el tiempo.

19 En el 2007 cuando se inicia el ejercicio de formulación de los planes locales de SAN en los municipios, se promueve la conformación de la red local como escenario de participación articulado a los Consejos de Política Social u otras instancias de participación que se han creado en los municipios para la planificación y gestión del desarrollo. 
Con base en la experiencia obtenida en esta fase inicial de implementación del proyecto, puede decirse que procurar el mejoramiento de las condiciones de seguridad alimentaria en familias y comunidades es un propósito que recién empieza a incorporarse en el imaginario de los técnicos locales y de las familias y comunidades, pero son muchos los frentes que deben atenderse.

En primera instancia, la totalidad de los municipios consideran que para obtener seguridad alimentaria es suficiente con ofrecer semillas o animales para la cría (disponibilidad), como una manera de obtener proteína vegetal y animal para el consumo (acceso). Este foco descuida otros aspectos sustanciales como:

- Las características de tenencia y propiedad sobre la tierra de las familias (la mayoría de ellos viven de su fuerza de trabajo y no son dueños de los medios de producción) porque ello define las posibilidades concretas y los recursos de los que disponen para la producción;

- La conformación del grupo familiar y sus necesidades básicas de manutención, que establece formas organizativas y de participación diversas para el trabajo en el predio;

- La capacidad organizativa de la comunidad, que suele ser débil o escasa, no obstante se promueven formas de asociación o agrupación como requisito para acceder a los insumos para la producción, y no como posibilidad real para que las organizaciones de base se potencien en el trabajo comunitario y desde allí promuevan la autogestión conforme a sus requerimientos.

En segundo lugar, el volumen de producción para el autoconsumo familiar suele ser insuficiente para satisfacer la demanda de alimentos en cantidad y diversidad nutricional requerida, con pocas posibilidades de mantener la producción en el tiempo. Las familias y las comunidades no solo requieren producir alimentos, sino, fundamentalmente, requieren condiciones y oportunidades para que la alimentación (en perspectiva integral) sea adecuada: condiciones de trabajo, crédi- to, ingresos, disponibilidad de herramientas e insumos, comercialización, precios adecuados y sostenibles de los productos, mercados de comercialización, entre otros; son aspectos que deben contemplarse para obtener mayor integralidad en la atención de una situación que es multicausal.

En tercer lugar, respecto a los procesos educativos, es necesario avanzar en el conocimiento de las prácticas de alimentación que las familias y las comunidades poseen producto de las tradiciones y los aprendizajes sociofamiliares. No obstante, las instituciones y los profesionales buscan 'modificar' comportamientos desde los cánones nutricionales teóricamente validados, desconociendo los significados sociales que subyacen a la alimentación y al acto de alimentarse y que, por lo tanto, están incorporadas en las formas de vida de las personas. Luego, promover prácticas y hábitos adecuados de alimentación requiere como punto de partida conocer a las significaciones de las personas y desde allí impulsar formas de concientización respecto de las opciones o restricciones que sus prácticas ofrecen a su propio desarrollo.

Esta concepción exige voluntad política, tiempo y recursos; sin embargo, las administraciones municipales centran su interés en la cobertura lograda, mediante 'activismos' que poco favorecen cambios en los sujetos, pero que sirven para responder a las exigencias institucionales y políticas con las que se mide el desarrollo y la gestión local.

Finalmente, una de las mayores limitantes para la garantía del derecho a la alimentación reside en la debilidad institucional con la que opera el desarrollo en lo local, departamental y nacional que, además de compleja, es burocrática, lo que dificulta la respuesta oportuna a las lógicas y las necesidades de la población.

Esta debilidad institucional para la gestión de la política es una limitante porque, de un lado, no hay consensos entre los actores sociales respecto al tipo de desarrollo que se espera lograr, sino que los programas de gobierno obedecen a concepciones ideológicas 
que se imponen y a pretensiones particulares de los grupos que ostentan y ejercen el poder político local, lo que hace que la gestión del desarrollo se restrinja a intereses político partidistas, más que a intereses comunes y a la responsabilidad ética que exige el bienestar y el bien común.

De otro lado, y en consonancia con lo anterior, no existe un norte sobre los derechos y el bienestar que debe lograrse como finalidad teleológica y que debe mantenerse como un propósito de Estado, más allá del cuerpo ejecutivo que en determinado momento gobierne; de ahí que algunas políticas y programas sociales se posicionen como 'banderas' o modas que duran cierto tiempo, conforme a los discursos normativos que emergen en el contexto nacional e internacional, como lo es la Seguridad Alimentaria. En tal sentido, las instituciones emprenden acciones en esta materia para responder con el obligatorio cumplimiento de una norma, más que como una convicción acerca de los derechos que tenemos como seres humanos.

En general, puede decirse que las prácticas que caracterizan la gestión municipal respecto a la implementación de la política SAN son:

- Discontinuidad en los procesos, lo que genera desconfianza en la gente.

- Aunque en la totalidad de municipios trabajados se ha conformado la Red SAN, esta carece de formalización y se evidencia voluntad política para favorecer los procesos con las organizaciones comunitarias. A esto se suma la diversidad en el manejo de los tiempos y los diversos compromisos institucionales que se superponen al trabajo en Red.

- Aunque los gobiernos locales entregan las semillas, los trámites administrativos no se corresponden con las necesidades concretas de la población. Estos, además de ser lentos suelen exigir una serie de documentación y procesos que restringen o limitan las posibilidades de acceso de las familias rurales, sumadas a las ya existentes por la distancia geográfica de la cabecera municipal, donde se concentra la oferta institucional.

\section{Conclusiones}

La aplicación de la política de Seguridad Alimentaria en el departamento de Caldas no apunta a resolver los problemas estructurales del hambre, sino a mitigarla con ayudas alimentarias y programas productivos coyunturales y marginales. Alcanzar la soberanía alimentaria integral implica cambiar la concepción de la política y la forma como se aplica en las familias y comunidades afectadas por la pobreza y la desnutrición.

Los programas y proyectos que se adelantan en las localidades no logran el propósito de garantizar la seguridad alimentaria, conservar y manejar adecuadamente los recursos naturales y lograr la equidad social, pilares del bienestar y el desarrollo humano de la población más vulnerable.

La seguridad alimentaria y nutricional de una población es un asunto que rebasa la disponibilidad de alimentos a través de los mecanismos del mercado y las políticas de ayuda alimentaria. Desde la titularidad de los derechos, esta es un asunto complejo, multicausal y multidimensional, que tiene que ver con los procesos y dinámicas familiares, tanto en el ámbito doméstico (producción, manejo, preparación y consumo) como en el público (diseño y ejecución de las políticas públicas); la gestión pública, los derechos económicos y las libertades individuales y familiares.

La transformación de la política de seguridad alimentaria, el manejo adecuado de los recursos y la equidad social se pueden superar en la medida en que las familias y las comunidades "tomen conciencia" de su situación y desde sus acciones cotidianas generen cambios en los ámbitos familiar, comunitario y gubernamental, espacios de decisión y acción fundamentales en la transformación de la realidad.

\section{Bibliografía}

CONPES. (2008). Documento No. 113, Política de Seguridad Alimentaria y Nutricional.

Comisión de Derechos Humanos. (2007). Organización de las Naciones Unidas. Disponible en: http://www. fidh.org/IMG/article_PDF/ El-derecho-a-la-alimentacion-en.pdf. Consultado octubre 16 de 2009. 
Cuadernos de Ceam (2004). Derecho a la alimentación en el Brasil de Lula. UnB Universidad de Brasilia. Centro de Estudios Avançado Multidisciplinares, NEAGRI, Núcleo de Estudios Agrarios. Cuaderno IV, No. 13. Maio 2004. ISSN 0103-510X.

DANE. (2005). Censo General de Población. www.dane.gov.co. Consultado el 16 de octubre de 2009.

FAO. (2009). El Estado de la Inseguridad Alimentaria en el mundo 2009. Crisis económicas: repercusiones y enseñanzas extraídas. Disponible en http://www.fao.org/ docrep/012/i0876s/i0876s00.htm, consultado octubre 16 de 2009.

FAO. (2002). Inseguridad Alimentaria: La población se ve obligada a convivir con el ham- bre y teme morir de inanición. El Estado de la Seguridad Alimentaria en el mundo.

Foro Social Mundial: Otro Mundo es posible. (2004). Brasil.

Merchán Mesón, Carlota (2007). Derecho a la alimentación y a la salud reproductiva desde la perspectiva de los derechos económicos, sociales y culturales. Prosalus. BITS $N^{\circ} 13$. Boletín Informativo de Trabajo Social - ISSN 1578-9578. E.U. de Trabajo Social de Cuenca, Universidad de Castilla-La Mancha

SEN, Amartya. (2002). El derecho a no tener hambre. Estudios de Filosofía y Derecho No. 3. U Externado de Colombia. Bogotá . (1999). Desarrollo y Libertad. Editorial Planeta Colombiana S.A. 


\section{Anexo $\mathrm{N}^{\circ} 1$}

Ubicación Geográfica del Departamento de Caldas

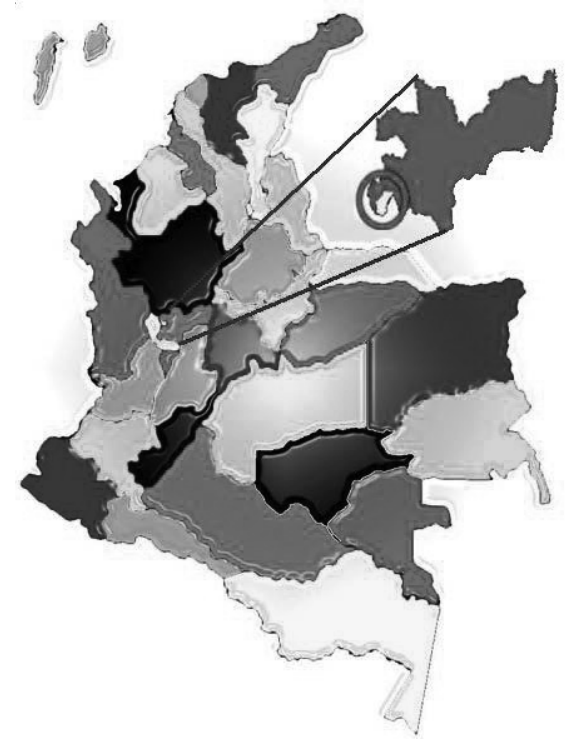

Anexo $\mathrm{N}^{\circ} 2$

Municipios de Caldas atendidos por el proyecto Fortalecimiento del capital social y humano para la seguridad alimentaria, en el segundo semestre de 2008

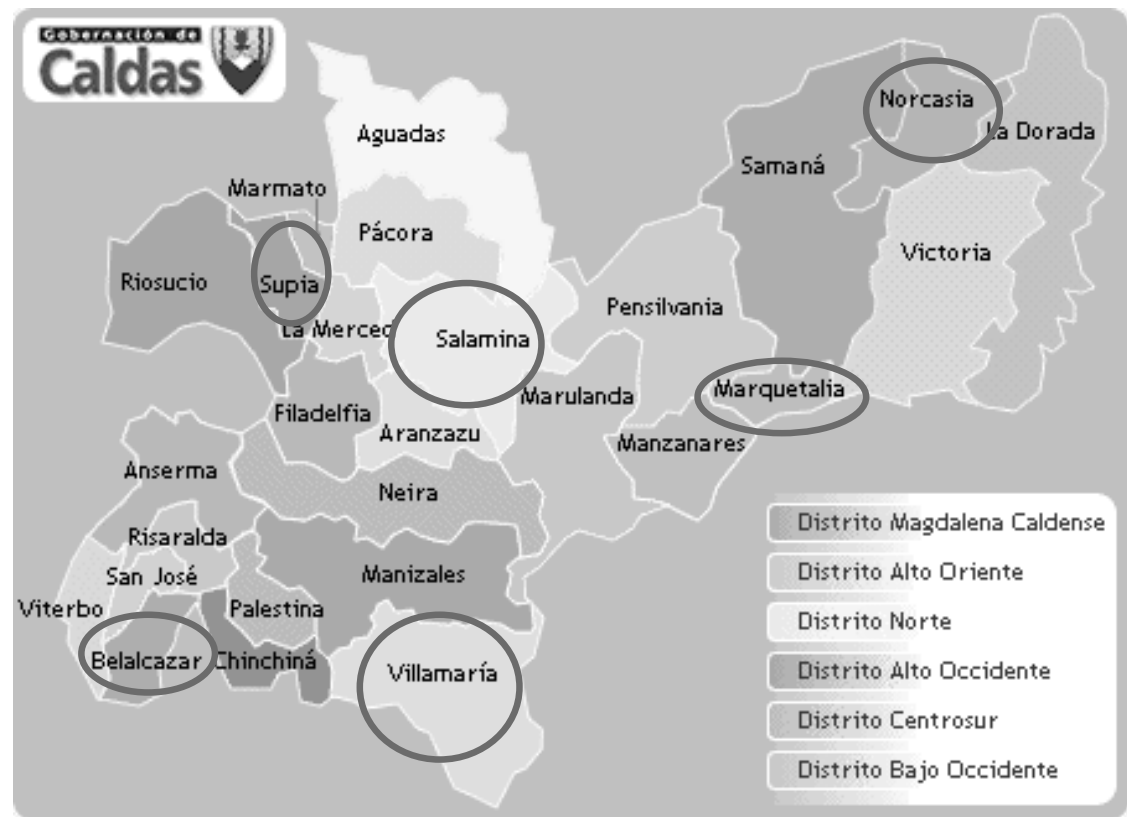

